\title{
Current Therapy in Inflammatory Bowel Disease: Why and How We Need to Change?
}

\begin{abstract}
Authors:
Katherine A Falloon, ${ }^{*}$ Claudio Fiocchi ${ }^{1,2}$

1. Department of Gastroenterology, Hepatology and Nutrition, Digestive Disease and Surgery Institute, Cleveland Clinic, Ohio, USA

2. Department of Inflammation \& Immunity, Lerner Research Institute, Cleveland Clinic, Ohio, USA

*Correspondence to fiocchc@ccf.org

Disclosure: $\quad$ The authors have declared no conflicts of interest.

Received: $\quad 18.06 .21$

Accepted: $\quad 10.09 .21$

Keywords: $\quad$ Artificial intelligence (AI), Crohn's disease (CD), inflammatory bowel disease (IBD), interactome, network medicine, omics, systems biology, ulcerative colitis (UC).

Citation:

EMJ Innov. 2021;6[1]:40-49.

During the last few decades, major progress has been made in the treatment of the two major forms of inflammatory bowel disease (IBD): Crohn's disease and ulcerative colitis. However, the success of the most advanced forms of therapy is at best 50\%, and most patients lose responsiveness with time and need to switch to alternative medications. This denotes that a therapeutic ceiling has been reached and brand new approaches are badly needed. This commentary first briefly reviews current and recent therapeutic approaches for Crohn's disease and ulcerative colitis, pointing out their limitations. This is followed by an objective evidence-based discussion of why the current approaches are far from optimal, and the commentary is concluded by proposing how to change IBD treatment based on the holistic concept of network medicine, and how to implement precision medicine for IBD using artificial intelligence-based multi-omics analyses.
\end{abstract}

\section{Abstract}

\section{INTRODUCTION}

Inflammatory bowel disease (IBD) represents a group of idiopathic chronic inflammatory disorders of the gastrointestinal tract, including Crohn's disease (CD), ulcerative colitis (UC), and IBD unspecified. The growing prevalence and significant impact of IBD around the world has made it the focus of decades of dedicated investigation, but the understanding of its pathogenesis has remained disappointingly elusive.' The so called 'hygiene hypothesis' has been proposed, based on the notion that increased industrialisation, better sanitation,

and subsequent societal changes could explain the worldwide emergence of IBD. ${ }^{2}$ However, the combined interplay of environmental, social, genetic, epigenetic, transcriptional, microbial, dietary, and immune factors that lead to the development of IBD and dictate its clinical course is poorly understood. ${ }^{3}$

Currently, the diagnosis of IBD is still made via expert assessment based on a combination of symptoms, laboratory parameters, stool testing, imaging, endoscopic, and histologic evaluation of the gastrointestinal tract. ${ }^{4,5}$ The result of this traditional approach is not always straightforward. The diagnosis of IBD can be 
delayed for years and, once made, may require reclassification into a different subcategory. ${ }^{6}$ As many as $10 \%$ of patients diagnosed with UC ultimately end up being reclassified as CD, while $5 \%$ of those labelled with CD ultimately end up with their diagnosis being reclassified as UC. ${ }^{7,8}$ This difficulty in classification is especially evident in patients who have undergone what was once touted as a curative colectomy for UC, followed by restorative pouch creation. In fact, as many as $40 \%$ of these patients go on to develop recurrent symptoms, and 10\% are diagnosed with CD-like disease of the pouch, making the distinction between UC and CD more fluid than once thought. 9,10

Regardless of IBD type, the clinical course is highly heterogeneous. Some patients have a benign course, some have relapsing disease, and some present with an aggressive phenotype complicated by strictures, fistulas, perianal disease, dysplasia, malignancy, and/ or extra-intestinal manifestations." As a result of this variability and diagnostic delay, many patients with IBD will have one or more of these complications prior to when therapy is initiated. ${ }^{12}$

Thus, considering all the above obstacles, it is not surprising that, despite the wide and growing number of therapeutic agents approved for UC or $\mathrm{CD}$, therapeutic efficacy remains impossible to predict and regrettably modest.

\section{CURRENT THERAPEUTIC PARADIGMS}

\section{Early Immunomodulators}

The first drug accidentally shown to have a therapeutic effect in UC was salazopyrin (a salicylate compound) in the late 1940s. ${ }^{13}$ Since that time, a number of 5-amino salicylic acid (5-ASA) formulations have been introduced in both oral and topical forms. The intestinal anti-inflammatory action of these agents is multifactorial and still poorly understood but is thought to be mediated via modulation of prostaglandin synthesis and suppression of pro-inflammatory cytokines. ${ }^{14}$ Once the only therapeutic option for IBD, 5-ASA compounds are now recommended in a relatively small subset of patients with mild to moderate UC, but not in patients with severe UC or CD. 4,5
Corticosteroids were the next agents to enter the IBD armamentarium. By binding cytoplasmic DNA receptors to inhibit DNA synthesis and tamp down the inflammatory response, they once became the mainstay of IBD therapy..$^{15}$ However, a solid body of evidence has established that corticosteroids are useful primarily for initiating but not maintaining remission, and display a number of side effects, ranging from metabolic derangements to psychiatric disturbances. ${ }^{4,5}$ Budesonide, a steroid with some degree of intestinal specificity, has fewer side effects, but is still unable to maintain remission, and is less effective than systemic steroids. ${ }^{16}$

Drugs with immunomodulatory activity have been used for cancer treatment since the early 1960's, but their use for IBD started only after that of corticosteroids. Azathioprine and 6-mercaptopurine antagonise purine metabolism to deactivate $\mathrm{T}$ lymphocytes and suppress the immune system, but exhibit a long, delayed therapeutic action. ${ }^{4,5,14}$ Only suitable as maintenance therapy, they are currently seldom used alone given their limited efficacy and substantial side effect profile, which includes dose-dependent myelosuppression, hepatotoxicity, rash, and malignancies, especially non-Hodgkin's lymphoma and nonmelanomatous skin cancers. ${ }^{4,5,17}$ Methotrexate, which blocks immune cell proliferation and induces immune cell apoptosis, is only useful in the induction and maintenance of remission of CD and is also seldom used alone. 4,18 Cyclosporine and tacrolimus are additional immunomodulators rarely used today due to modest efficacy and unfavourable collateral effects.

\section{Antibiotics, Prebiotics, and Probiotics}

During the 1980 s and into the early 2000s, antibiotics became another mainstay of therapy. Many of them were tried, including rifaximin, clarithromycin, metronidazole, ciprofloxacin, amoxicillin, tetracyclines, vancomycin, and various combinations of these agents..$^{19}$ Although their use is theoretically justifiable, several randomised clinical trials showed trivial to minor benefits and no prolonged effect. Antibiotics are no longer routinely used in UC or CD except in selective clinical settings as, for example, therapy for pouchitis, adjunctive therapy for perianal $C D$, or prevention of post-operative $C D$ recurrence, though research on the role of antibiotics in IBD is 
still ongoing. ${ }^{20}$ The use of prebiotics and probiotics is another area of ongoing research, especially given their favourable safety profile. These agents have shown some promise (especially $V S L \# 3{ }^{\circledR}$ [Alfasigma USA, Inc., Covington, Los Angeles, USA] in pouchitis), but studies are limited and have not yet convincingly demonstrated a robust benefit.21,22

\section{Biologics}

The current mainstay of IBD therapy is biological therapy, which is primarily based on monoclonal antibodies directed against specific molecules with immunomodulatory or inflammatory activity. Biologics are divided into four classes based on mechanism of action. The first and largest class of biologics are agents targeting TNF, a pro-inflammatory cytokine found in increased concentrations in the blood, stool, and colonic mucosa of patients with IBD. ${ }^{23-25}$ Infliximab, the first of these agents, is administered intravenously and is used in patients with both UC and CD. Although infliximab revolutionised the care of patients with IBD and is among the ten highest grossing drugs in the USA, it maintains clinical remission in only $25 \%$ of patients with CD and $35 \%$ of patients with UC at 54 weeks. ${ }^{26,27}$ Biosimilars targeting TNF have since been introduced and are of lower cost but still of similar efficacy.

Adalimumab, a successor of infliximab administered in a subcutaneous fashion, has similar remission rates of only $25 \%$ at Week 54 in patients with CD, and $17 \%$ at Week 52 in patients with UC. ${ }^{28-30}$ Clinical trials with subsequent anti-TNF agents (golimumab, which has only been approved for UC; and certolizumab, which has been approved only for CD) did not fare much better, whether endpoints were clinical remission or mucosal healing. ${ }^{31,32}$ To help overcome loss of response, anti-TNFs are often combined with immunomodulators, and therapeutic drug monitoring is being increasingly utilised, but even so many patients fail anti-TNF therapy. ${ }^{33}$ In addition, these agents also carry side effects, ranging from infusion reactions, to opportunistic infections, to possible increased risk of malignancy, especially when used in combination with azathioprine.

A different type of biologic is the anti-leukocyte trafficking agent vedolizumab, engineered to block a4ß7, an integrin mediating migration of immune cells from the peripheral blood into the intestinal tract.14 Though this gut specificity makes for an improved side effect profile when compared to the anti-TNFs, remission rates are still limited, with $42 \%$ of patients with UC and $32 \%$ of patients with CD in remission at 52 weeks. ${ }^{34,35}$

Another type of biologic is ustekinumab, which targets the common p40 subunit of receptors for IL-12 and $23^{14}$ cytokines, which induce the production of the cytokines interferon- $y$ and IL-17, respectively. This target was identified through genome-wide association studies and subsequently confirmed to be involved in $C D$ pathogenesis. ${ }^{14}$ However, overall ustekinumabinduced remission rate is still only around 53\% in CD and $44 \%$ in UC at 52 weeks. ${ }^{36,37}$ Its safety profile is comparable to that of vedolizumab.

Taken all together, response rates to biologics range anywhere from $20-50 \% .{ }^{38,39}$ When the high placebo response rates (anywhere from 7-30\%) in drug trials are factored in, it becomes clear that, although a number of treatment options are now available, none can be considered a 'magic bullet'. In addition, even among initial responders, as many as $40 \%$ will lose response over time, ${ }^{40}$ and those who lost response to one biologic agent are less likely to respond to a second. Moreover, despite biologics, around $11 \%$ of patients with UC will still require colectomy due to medically refractory disease, acute severe ulcerative colitis, mucosal dysplasia, or malignancy, and up to $70 \%$ of patients with $C D$ will require surgery due to medically refractory disease, or complications that respond poorly to medical management. ${ }^{41,42}$

\section{RECENT THERAPEUTIC PARADIGMS}

\section{New Agents and Combination Therapy}

There are currently numerous new agents in Phase II and III clinical trials, including new monoclonal antibodies and new small molecules. Some of the new antibodies target the p19 subunit of the IL-23 receptor, with several agents under investigation, including risankizumab, brazikumab, and guselkumab. ${ }^{43}$

A number of small molecules have been developed that are either approved or under active investigation. These orally administered agents block JAKs, a family of pro-inflammatory enzymes that activate multiple immune pathways 
instead of a single cytokine or receptor. The only JAK inhibitor presently approved is tofacitinib, but this agent is only effective in $U C$ and response rates at 52 weeks are $34-41 \%$ depending on dosage, with a less favourable safety profile compared to vedolizumab or ustekinumab. ${ }^{44,45}$ Other JAK inhibitors are under investigation and development and include upadacitinib, filgotinib, brepocitinib, peficitinib, TD-1473, and Pf-06651600/Pf-06700841, but it is unlikely that their therapeutic efficacy will be substantially better. ${ }^{46}$ Another different type of small molecule under study is AVX-470, a new anti-TNF oral formulation. ${ }^{47}$

Inhibitors of lymphocyte trafficking agents with a mechanism of action similar to vedolizumab are also under investigation, such as abrilumab, etrolizumab, ontamalimumab, and AJM300.45 In addition, one agent targeting leukocyte trafficking via sphingosine-1-phosphate, named ozanimod, was newly approved by the U.S. Food and Drug Administration (FDA) for UC, and other agents in this class are in Phase II and III trials. ${ }^{48}$

Given that all these agents are targeting similar pathways, the chance that any of them will have a significantly improved efficacy compared to available agents is low. In addition, real life effectiveness of all drugs on the market is extremely low, ranging from $5-13 \%, 49$ so promise and potential are limited. Partly for these reasons, there is an emerging interest in combination therapy, in which two biologics with different mechanisms of action are combined. The field is still in its infancy, but early data suggest response rates remain at best around 50\%. . $0,51^{2}$

\section{ALTERNATIVE AND COMPLEMENTARY APPROACHES}

Looking at the history of IBD therapeutics, it is obvious that the immune system has been the single major focus of therapeutic interventions, even though IBD is a multifactorial disease. In fact, apart from the encouragement of smoking cessation, there has been a limited attention to any treatments that do not involve modulation of the immune system. Elemental diets have shown some promise, but are difficult for patients to tolerate. ${ }^{52}$ There is also some evidence for benefits from exclusion diet (excluding wheat, dairy, emulsifiers, maltodextrins, carrageenans, and sulfites, and is low in animal fat), or a partial elemental diet (a free diet along with a minimum $900 \mathrm{kcal} /$ day of elemental nutrition) in patients with CD. ${ }^{52}$ Recently, stem cell therapies have also emerged as possible therapeutics for perianal $C D$, though the work is preliminary and response rates are thus far similar to that of biologics. ${ }^{53}$ Finally, faecal microbial transplantation shows some promise, but it is still under study and has not convincingly emerged as a reliable treatment alternative. ${ }^{54}$

\section{MEDICALLY REFRACTORY DISEASE}

It is important to note that there are a number of specific clinical presentations of IBD, which will not respond well to any of the above therapies. Perianal and fistulising diseases almost always require surgical intervention, as does fibrostenotic disease. Once dysplasia and/or malignancy develops, the vast majority of patients will also require either advanced endoscopic or surgical interventions.

\section{WHY TO CHANGE THE THERAPEUTIC APPROACH TO INFLAMMATORY BOWEL DISEASE}

Taking all the above information into consideration and honestly judging how good current IBD therapies truly are, one must reach the obvious conclusion that they are not particularly effective. This brings up the existential issue of why IBD cannot be cured, and the answer is that IBD cannot be cured because 'life is complicated' 55 i.e., 'IBD is complicated'. In fact, both CD and UC are believed to be the result of the interaction of four major components, or -omes: the exposome (the external surrounding and internal body environment), the genome (the host's genetic makeup), the microbiome (the bacteria, fungi, viruses, and archaea harboured in the gut), and the immunome (the systemic and mucosal immune systems).

The exposome is exceedingly complex, changes continuously because of human intervention that makes it totally unpredictable, is impractical to qualify, and hard to quantify. The genome is structurally stable, individualised and qualifiable, but its function depends on gene-gene interactions and the exposome. The microbiome 
is also extremely complex and individualised, varies continuously due to ingestion of food components, antibiotics, xenobiotics, and drugs; is hard to quantify and qualify; and is both exposome- and genome-dependent. The immunome is highly complex, constantly and variably adapting to immune and metabolic signals, is hard to quantify and only partially qualifiable, and is exposome-, genome- and microbiome-dependent. Each of these four factors comprises countless components and performs incalculable interactions, so that the resulting outcome (the IBD interactome) is variable, as it depends on the reciprocal and interdependent function of each -ome. ${ }^{56}$ This leads to a more realistic definition IBD: IBD is a life-long, heterogeneous, and highly complex disease that cannot be cured by targeting one single disease component at any one time.

In addition, it should not be forgotten that humans are like snowflakes, 'no two are alike', and that the components of IBD are different and act differently in each patient with $C D$ or UC. Each patient is exposed to and lives in a particular corner of the world, creating a distinct exposome, the genome is determined at the time of conception, and the gut microbiome results from a myriad of dietary and other factors that start right after birth and act for the rest of one's life. Consequently, when an IBD factor initiates the chain of events that will eventually result in IBD, each patient will use different receptors, activate different signalling molecules, bind different DNA loci, express and transcribe different genes, translate different proteins, and produce distinct pro-inflammatory products. These products may differ, but all will induce a damaging inflammatory process that will be recognised as IBD at the clinical level (Figure 1). So, if IBD is so complex, let's embrace its complexity to try to better understand it and look for different means to treat it. ${ }^{57}$

An initial consideration in IBD treatment is to ask the question: "What is being treated? Is the whole disease being treated, or simply the inflammation, which is the response to the disease?" About two decades ago, Carl Nathan wrote: "When primary pathogenetic events are unknown, control of inflammation is sometimes the next best option." 58 Since then, a lot of progress has been made in understanding pathogenic events in IBD, but healthcare professionals still adopt control of inflammation as the primary therapeutic approach for IBD. Indeed, when healthcare professionals consider the large variety of therapeutic options described above, it is obvious that current IBD therapies are utterly lopsided. There are no genetic therapies for IBD but, even if there were, they are unlikely to offer any significant benefit as genes alone do not cause IBD. Healthcare professionals can try to intervene in the exposome and change the patient's environment with lifestyle modifications such as diets and smoking cessation, which are predictably poorly accepted and hard to implement. Healthcare professionals can also modulate the microbiome via diets and administering antibiotics, probiotics, or faecal microbiota transplantation, but the results are incomplete and of short duration, as pointed out above. So, what is left is to target intestinal inflammation by the numerous and variable means mentioned above: 5-ASA compounds, corticosteroids, immunosuppressors, anticytokines, anti-chemokines, anti-receptors, anti-integrins, anti-signalling molecules, sphingosine-1-phosphate receptor agonists, leukapheresis, bone marrow transplants, and stem cell transplants. Combined, they represent roughly $90 \%$ of what can offer objective benefits to the patients, regardless of side effects and anticipated loss of efficacy (Figure 2). ${ }^{59}$ As indicated above, the best response rates are still around $50 \%$ or less. ${ }^{50}$ This unsatisfactory condition results directly from the single target-single medicine approach, and has remained unchanged for decades, indicating that a therapeutic ceiling has been reached, no matter what medication is prescribed..$^{40}$ Thus, it seems obvious that progress has stalled and innovative approaches to IBD therapy are needed.

\section{HOW TO CHANGE THE THERAPEUTIC APPROACH TO INFLAMMATORY BOWEL DISEASE}

If the complexity of IBD is accepted, and the fact that the single target-single medicine approach has stalled progress, one solution is to think about IBD in a completely different way. If IBD is complex, healthcare professionals should embrace its complexity: IBD has multiple components with multiple links, 
comparable to what happens in most networks (social, financial, marketing, political, business, computer, etc.). Thinking in terms of an 'IBD network', a 'network medicine' approach should be applied. Network medicine is based on the principle that "rather than trying to force disease pathogenesis into a reductionist model, network medicine embraces the complexity of multiple influences on disease and relies on many different types of networks." 60 Intrinsic to the notion of network medicine is integration that, in the case of IBD, means to incorporate all pathogenic components into a unifying functional entity, the IBD interactome. ${ }^{56}$ Interactomes are formed by innumerous biological components, but a series of technological tools are now available that can help to dissect complex biological systems, like transcriptomics, proteomics, epigenomics, single cell omics, and others, and all can be analysed and unified by computational modelling. ${ }^{61}$ This approach generates what has come to be known as 'big data', i.e., a massive amount of information that can only be interpreted with the help of

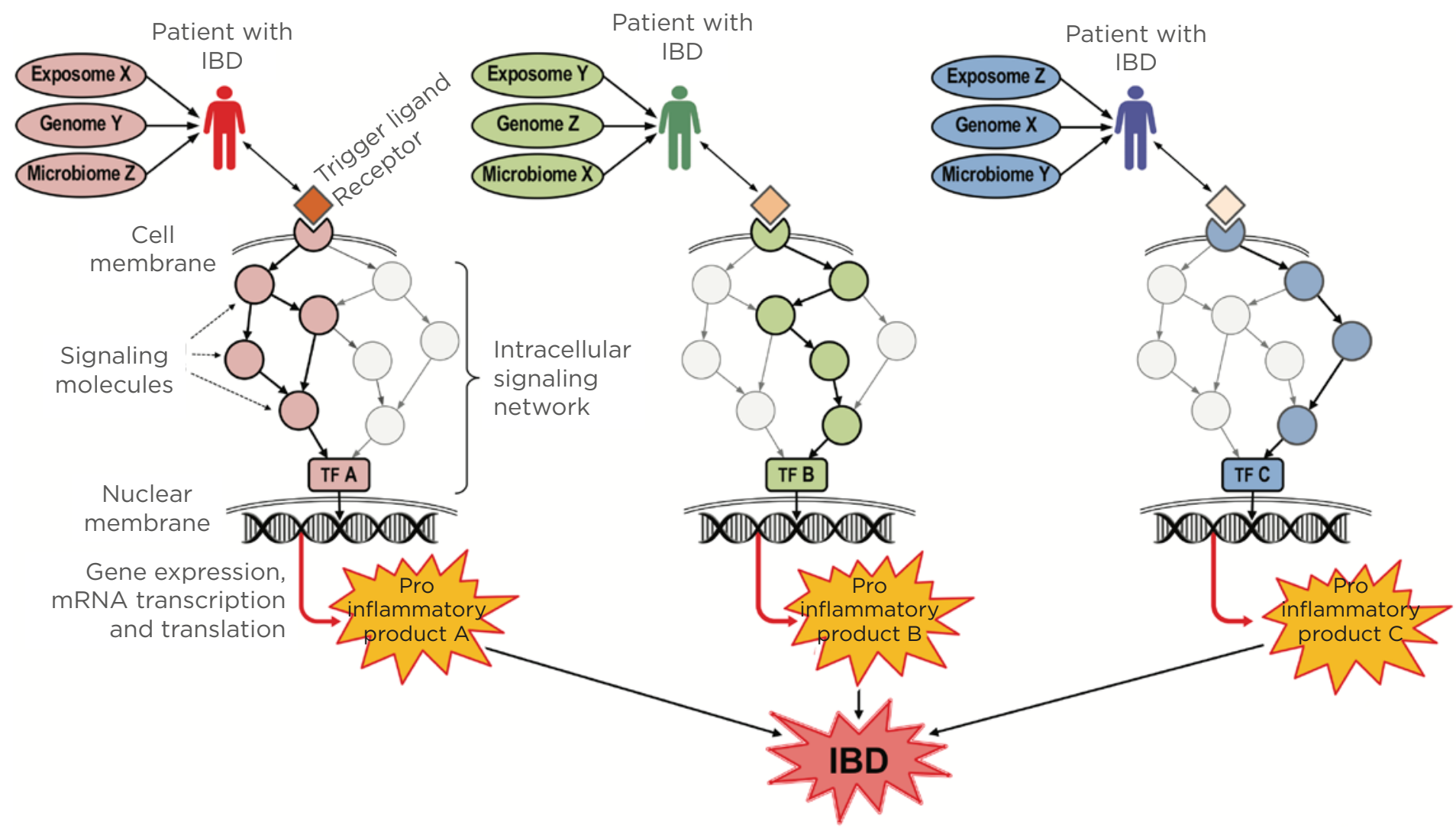

Figure 1: Schematic representation of how patients with inflammatory bowel disease use different pathogenic mechanisms that result in the same organotypic expression (intestinal inflammation).

All three patients (the red, green, and blue) were conditioned by the same key factors responsible for disease predisposition and expression, i.e., the exposome, the genome, and the microbiome. However, each of these conditioning factors differ in each patient (red patient: exposome X, genome $Y$, and microbiome Z; green patient: exposome $\mathrm{Y}$, genome $Z$, and microbiome $X$; and blue patient: exposome $Z$, genome $X$, and microbiome $Y$ ). Consequently, when the disease is triggered by the binding of various ligands (drugs, chemicals, pollutants, toxins, food components, metabolites, hormones, microbes, etc.) to their cell receptors, distinct signalling and activation molecules are engaged in each patient, creating intracellular signalling networks that are unique to the red, green, and blue patients. As a result, each signalling network leads to the activation of distinct TFs ( $A, B$, and $C$ ) that, once translocated into the nucleus, induce the expression of diverse inflammatory genes. Each gene is transcribed into mRNA and eventually translated into a particular pro-inflammatory product ( $A, B$, and $C$ ) such as the cytokines TNF, IL-1, IL-6, IL-17, etc. that are secreted into the intestinal tissue microenvironment. Although each cytokine is unique, they all induce pro-inflammatory events, resulting in a largely non-specific form of inflammation with effects and manifestations that are clinically recognised as IBD.

IBD: inflammatory bowel disease; mRNA: messenger RNA; TF: transcription factor. 


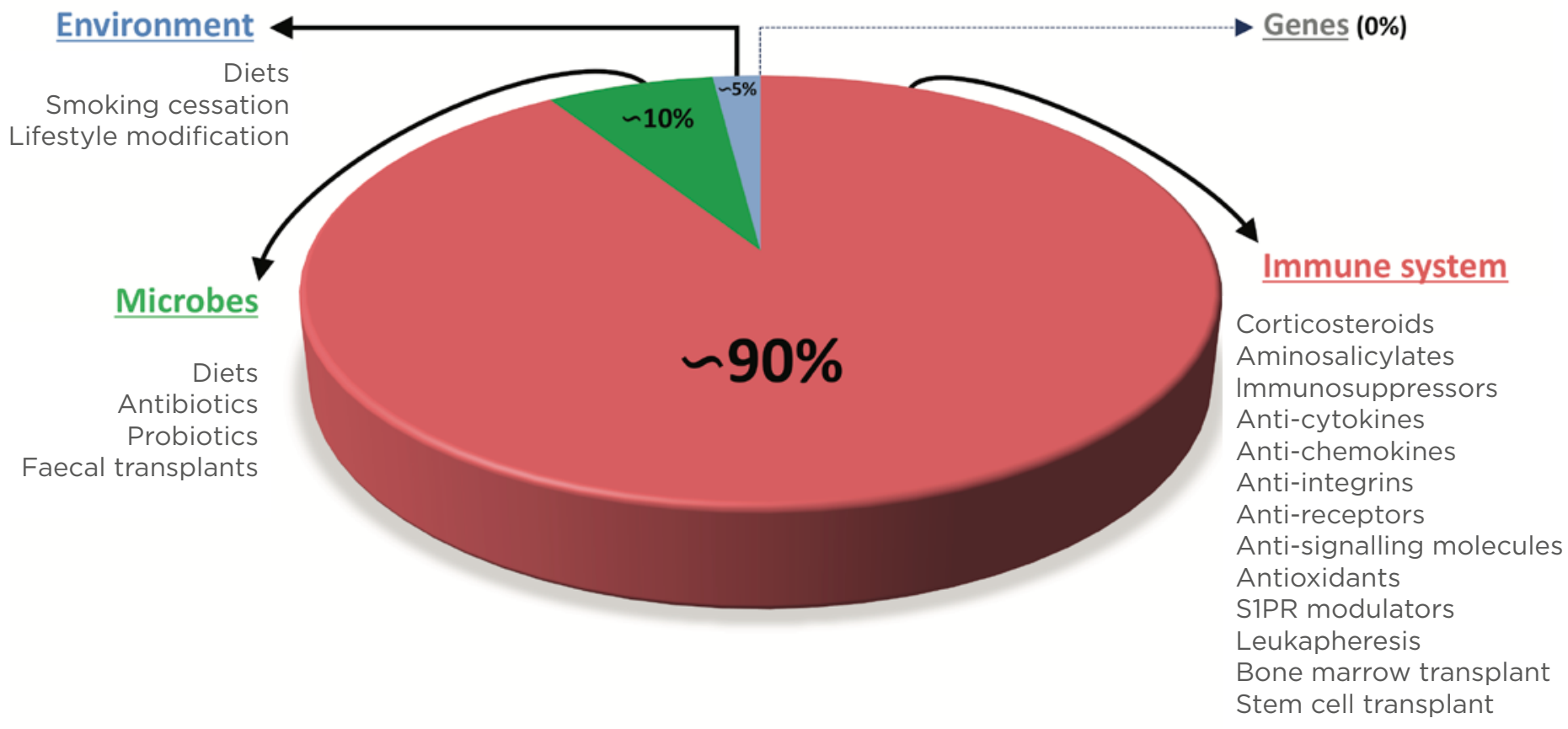

Figure 2: Relative proportion of the different therapeutic interventions available to treat inflammatory bowel disease by targeting the four major pathogenic factors.

There is no genetic therapy for CD or UC. Intervention on environmental factors can be attempted with diets, smoking cessation, and lifestyle modification but represents a tiny (approximately 5\%) proportion of all treatment modalities. Modulation of the gut microbiota is approximately $10 \%$ of all possible therapeutic intervention. The immune system is the dominant (approximately 90\%) form of treatment for IBD and can be accomplished with a large number of small molecules, biologics, and cell transplants.

CD: Crohn's disease; IBD: inflammatory bowel disease; S1PR: sphingosine-1-phosphate receptor; UC: ulcerative colitis.

machine learning and other types of artificial intelligence $(\mathrm{Al}){ }^{62,63}$

At this point, it is useful to establish an analogy between a network system and the IBD network (interactome). All networks have a 'hub', a dominant component that is usually centrally located and controls the function of all the peripheral components of the network. If the hub is disabled, the whole network is disrupted and ceases to function, being practically eliminated. Biological networks, including disease networks, function the same way, with central hubs and peripheral components, ${ }^{64}$ and if the hub is disabled the disease is eliminated. Applying these concepts to the IBD network, there is a network composed of genes, environmental factors, microbes, immune cells, and other elements (Figure 3A). When healthcare professionals intervene at the periphery of the network, like currently done with antibiotics, immunosuppressants, and biologics, there have been some effect of variable degree, but the best effect is obtained when the disease hub is targeted (Figure 3B). If targeting of the disease hub is completely successful, then the whole disease network is disabled, and the disease is eliminated (Figure 3C).

To implement this strategy, the following steps are carried out: biosamples (blood, serum, tissues, stools, etc.) from patients with IBD and active and inactive disease as well as healthy controls are subjected to multi-omic analyses (genome, epigenome, transcriptome, microbiome, etc.); the respective molecular networks of each ome are identified; Al-based systems, such as machine-learning, integrate the different omic data; analysis of the integrated omic data generates the IBD network and identifies the hub of each network; the identified hub becomes the molecule to be therapeutically targeted; the biological relevance of the target to IBD is confirmed with biochemical, cellular, and animal studies; a computational docking platform to the crystal structure of the target is used to generate 


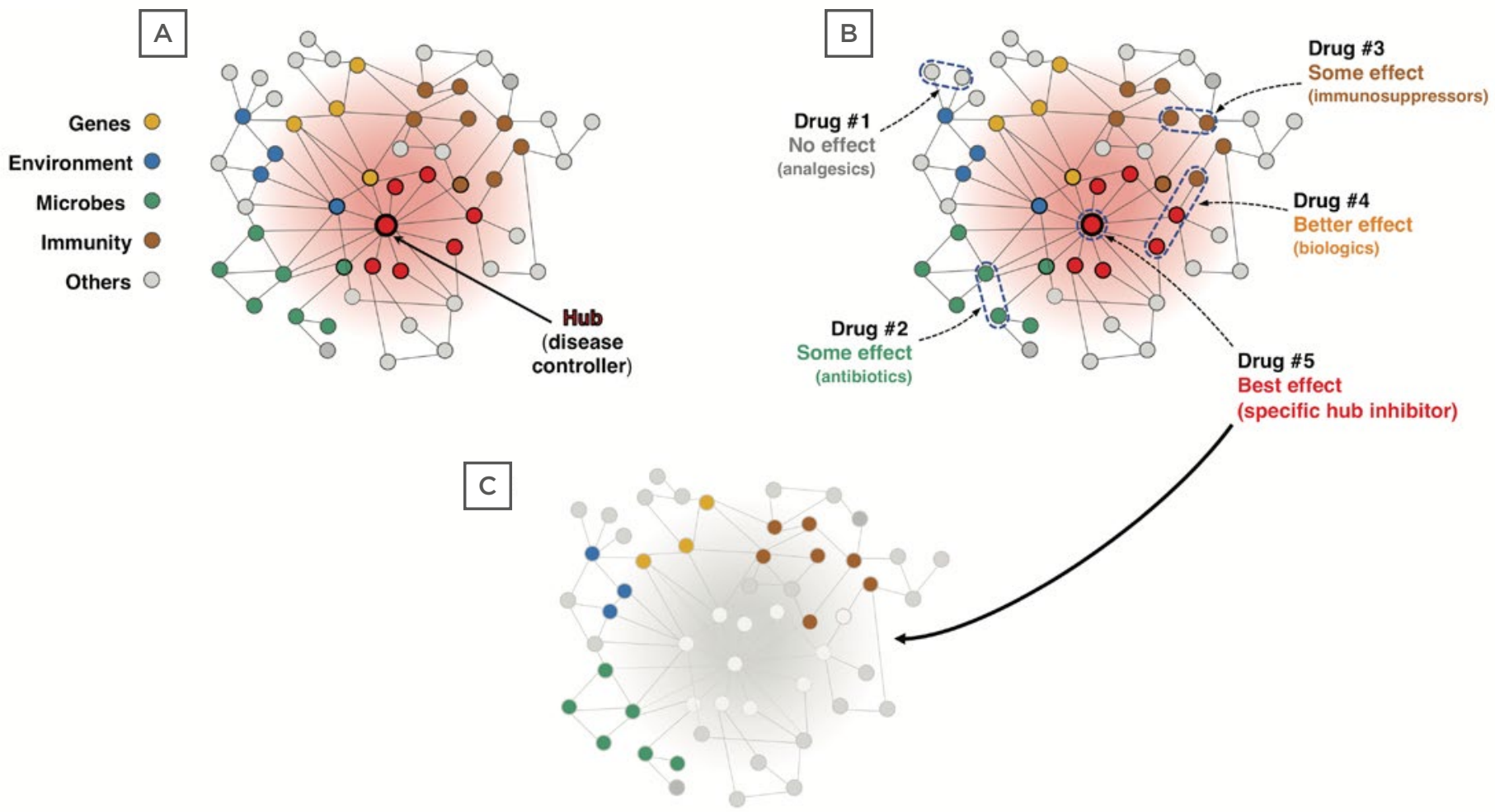

Figure 3: Graphical representation of the inflammatory bowel disease network and the effect of various therapeutic approaches.

A) The IBD network is composed of genes, environmental factors, microbes, immune cells, and other elements, and is controlled by a central hub, which can be a gene, a protein, a metabolite, a microbial product, a signalling molecule, an enzyme, etc. B) When a different way to try to disrupt the network is used, the results will vary depending on what part of the network is targeted. Drug \#1 (analgesics) acts at the extreme periphery of the network and will have no effect; Drug \#2 (antibiotics) acts on peripheral microbial components and may offer some benefit; Drug \#3 (immunosuppressors) acts on immune components also peripherally located and will induce a partial response; Drug \#4 (biologics) acts on some immune components plus other components more centrally located and will afford a better effect; and Drug \#5 (specific hub inhibitor) will have the best effect by inhibiting the centrally located disease hub. C) Once the disease hub is disabled the whole disease network is disrupted and IBD is structurally and functionally eradicated.

IBD: inflammatory bowel disease.

small molecules inhibitors; the inhibitors are tested for drug-drug interactions, toxicity, and safety, and the overall best is clinically tested first in healthy volunteers and subsequently in patients. The newly identified drug will not be effective in all patients with IBD, but only those whose disease interactome is controlled by the specific hub against which the new drug was developed. With this strategy, different molecular subgroups of patients with IBD will be categorised, and each molecularly homogeneous subgroup will receive a specific drug that will target the hub controlling the disease in that subgroup. ${ }^{65}$
This omic- and Al-based approach will drastically change the way IBD is treated as each patient will receive drugs specifically designed for their underlying mechanism of disease, unlike drugs that broadly block mechanisms of inflammation. This approach fosters and speeds up the ongoing transition period between the old and the new. The old is the traditional physician-based approach based on classical medical tools (history, physical exam, blood chemistry, serology, stools exam, imaging, endoscopy, biopsy, and histology), that diagnoses and classifies patients with IBD based on traditional clinical phenotypes, and non-disease specific anti-inflammatory 
drugs end up being prescribed. The new Albased network approach to IBD therapy is not purely hypothetical but is actually under active development, with the discovery and targeting of newfound hubs in specific IBD subgroups and allowing the implementation of precision medicine. ${ }^{66}$

\section{References}

1. Alatab S et al. The global, regional, and national burden of inflammatory bowel disease in 195 countries and territories, 1990-2017: a systematic analysis for the Global Burden of Disease Study 2017. Lancet Gastroenterol Hepatol. 2020;5(1):1730.

2. Baumgart D, Carding $\mathrm{S}$. Inflammatory bowel disease: cause and immunobiology. Lancet. 2007;369(9573):1627-40.

3. Fiocchi C, lliopoulos D. What's new in IBD therapy: An "omics network" approach. Pharmacol Res. 2020;159:104886.

4. Lichtenstein $\mathrm{G}$ et al. ACG clinical guideline: management of Crohn's disease in adults. Am J Gastroenterol. 2018;113(4):481-517.

5. Rubin D et al. ACG clinical guideline: ulcerative colitis in adults. Am J Gastroen-terol. 2019;114(3):384-413.

6. Blackwell J et al. Prevalence and duration of gastrointestinal symptoms before diagnosis of inflammatory bowel disease and predictors of timely specialist review: a population-based study. J Crohns Colitis. 2021;15(2):203-11.

7. Melmed $\mathrm{G}$ et al. Predicting a change in diagnosis from ulcerative colitis to Crohn's disease: a nested, casecontrol study. Clin Gastroenterol Hepatol. 2007;5(5):602-8.

8. Van Assche $\mathrm{G}$ et al. The second European evidence-based consensus on the diagnosis and management of Crohn's disease: definitions and diagnosis. J Crohn Colitis. 2010;4(1):727.

9. Ferrante $\mathrm{M}$ et al. Outcome after proctocolectomy with ileal pouchanal anastomosis for ulcerative colitis. Inflamm Bowel Dis. 2008;14(1):20-8.

10. Barnes $E$ et al. The incidence and definition of Crohn's disease of the pouch: a systematic review and meta-analysis. Inflamm Bowel Dis. 2019;25(9):1474-80.

11. Langhol E. Current trends in inflammatory bowel disease: the natural history. Therap Adv Gastroenterol. 2010;3(2):77-86.

12. Present $\mathrm{DH}$ et al. Infliximab for the treatment of fistulas in patients with Crohn's disease. N Engl J Med. 1999;340(18):1398-405.

13. Svartz M. The treatment of 124 cases of ulcerative colitis with salazopyrine and attempts of desensibilization in cases of hypersensitiveness to sulfa. Acta Med Scand. 1948;131(Suppl 206):465-72

14. Neurath MF. Current and emerging therapeutic targets for IBD. Nat Rev Gastroenterol Hepatol. 2017;14(5):269-78.

15. Ho GT et al. The efficacy of corticosteroid therapy in inflammatory bowel disease: analysis of a 5-year UK inception cohort. Aliment Pharmacol Ther. 2006;24(2):319-30.

16. Kuenzig M et al. Budesonide for maintenance of remission in Crohn's disease. Cochrane Database Syst Rev. 2014(8):CDO02923.

17. Chande $\mathrm{N}$ et al. Azathioprine or 6-mercaptopurine for maintenance of remission in Crohn's disease. Cochrane Database of Syst Rev. 2015(10):CD000067.

18. Feagan B et al. Methotrexate for the treatment of Crohn's Disease. The North American Crohn's Study Group. N Engl J Med. 1995;332(5):292-7.

19. Ledder O, Turner D. Antibiotics in IBD: Still a role in the biological era? Inflamm Bowel Dis. 2018;24(8):1676 88.

20. Townsend CM et al. Antibiotics for induction and maintenance of remission in Crohn's disease. Cochrane Database Syst Rev. 2019;2(2):CD012730.

21. Naseer $M$ et al. Prebiotics and probiotics in inflammatory bowel disease: where are we now and where are we going? Curr Clin Pharmacol. 2020;15(3):216-33.

22. Oliveira e Silva $\mathrm{N}$ et al. Probiotics in inflammatory bowel disease: does it work? World J Meta Anal. 2020;8(2):54-66

23. Braegger CP et al. Tumour necrosis factor alpha in stool as a marker of intestinal inflammation. Lancet. 1992;339(8785):89-91

24. Murch S et al. Location of tumour necrosis factor alpha by immunohistochemistry in chronic inflammatory bowel disease. Gut. 1993;34(12):1705-9.

25. Murch $\mathrm{S}$ et al. Serum concentrations of tumour necrosis factor alpha in childhood chronic inflammatory bowel disease. Gut. 1991;32(8):913-7.

26. Rutgeerts $P$ et al. Infliximab for induction and maintenance therapy for ulcerative colitis. N Engl J Med. 2005;353(23):2462-76.

27. Hanauer S et al. Maintenance infliximab for Crohn's disease: the ACCENT I randomized trial. Lancet. 2002;359(9317):1541-9.

28. Sandborn $W$ et al. Adalimumab fo maintenance treatment of Crohn's disease: results of the CLASSIC II trial. Gut. 2007;56(9):1232-9.

29. Colombel JF et al. Adalimumab for maintenance of clinical response and remission in patients with Crohn's disease: the CHARM trial. Gastroenterology. 2007;132(1):52-65.

30. Colombel J-F et al. Four-year maintenance treatment with adalimumab in patients with moderately to severely active ulcerative colitis: data from ULTRA 1, 2, and 3. Am J Gastroenterol. 2014;109(11):1771-80

31. Sandborn $\mathbf{W}$ et al. Subcutaneous golimumab maintains clinical response in patients with moderateto-severe ulcerative colitis. Gastroenterology. 2014;146(1):96-109.

32. Schreiber S et al. Maintenance Therapy with certolizumab pegol for Crohn's disease. N Engl J Med. 2007;357(3):239-50

33. Colombel JF et al. Infliximab, azathioprine, or combination therapy for Crohn's disease. N Engl J Med. 2010;362(15):1383-95.

34. Feagan BG et al. Vedolizumab as induction and maintenance therapy for ulcerative colitis. N Engl J Med. 2013;369(8):711-21.

35. Sands B et al. Vedolizumab as induction and maintenance therapy for Crohn's disease in patients naïve to or who have failed tumor necrosis factor antagonist therapy. Inflamm Bowel Dis. 2017;23(1):97-106.

36. Feagan B et al. Ustekinumab as induction and maintenance therapy for Crohn's disease. N Engl J Med. 2016;375(20):1946-60

37. Sands B et al. Ustekinumab as induction and maintenance therapy for ulcerative colitis. N Engl J Med. 2019;381(13):1201-14.

38. Alsoud D et al. Breaking the therapeutic ceiling in drug development in ulcerative colitis. Lancet Gastroenterol Hepatol. 2021;6(7):589-95.

39. Ford A et al. Efficacy of biologica therapies in inflammatory bowel 
disease: Systematic review and meta-analysis. Am J Gastroenterol. 2011;106(4):644-59.

40. Ben-Horin S, Chowers Y. Review article: loss of response to antiTNF treatments in Crohn's disease. Aliment Pharmacol Ther. 2011;33(9):987-95

41. Sedki M et al. Natural history of colectomy among hospitalized patients with ulcerative colitis in the contemporary era of biologics. Inflamm Bowel Dis. 2020;26(Suppl 1):S44.

42. Munkholm P et al. Intestinal cancer risk and mortality in patients with Crohn's disease. Gastroenterology. 1993;105(6):1716-23.

43. Hanžel J, D’Haens GR. Antiinterleukin-23 agents for the treatment of ulcerative colitis. Expert Opin Biol Ther. 2020;20(4):399-406.

44. Sandborn $W$ et al. Tofacitinib as induction and maintenance therapy for ulcerative colitis. N Engl J Med. 2017;376(18):1723-36

45. D'Amico $F$ et al. Tofacitinib in the treatment of ulcerative colitis: efficacy and safety from clinical trials to real-world experience. Therap Adv Gastroenterol. 2019;12:1756284819848631.

46. Fernández-Clotet $A$ et al. JAK Inhibition: The most promising agents in the IBD pipeline? Curr Pharm Des. 2019:25(1):32-40.

47. Harris $M$ et al. AVX-470, an orally delivered anti-tumour necrosis factor antibody for treatment of active ulcerative colitis: results of a first-in-human trial. J Crohns Colitis. 2016;10(6):631-40.
48. Misselwitz $\mathrm{B}$ et al. Emerging treatment options in inflammatory bowel disease: Janus kinases, stem cells, and more. Digestion. 2020;101(Suppl 1):69-82.

49. Wong $C$ et al. Estimation of clinical trial success rates and related parameters. Biostatistics. 2019;20(2):273-86

50. Alayo $Q$ et al. Safety and effectiveness of combining biologics and small molecules in IBD: systematic review with meta-analysis. 2021. Available at: https://papers. ssrn.com/sol3/papers.cfm?abstract id=3790447. Last accessed: 9 November 2021

51. Stalgis $C$ et al. Rational combination therapy to overcome the plateau of drug efficacy in inflammatory bowel disease. Gastroenterology. 2021;161(2):394-9.

52. Levine A et al. Evolving role of diet in the pathogenesis and treatment of inflammatory bowel diseases. Gut. 2018;67(9):1726-38.

53. Lightner AL. Stem cell therapy for inflammatory bowel disease. Clin Transl Gastroenterol. 2017;8(3):e82.

54. Levy A, Allegretti J. Insights into the role of fecal microbiota transplantation for the treatment of inflammatory bowel disease. Therap Adv Gastroenterol. 2019;12:1756284819836893.

55. Check Hayden E. Human genome at ten: life is complicated. Nature. 2010;464(7289):664-7.

56. de Souza HSP et al. The IBD interactome: an integrated view of aetiology, pathogenesis and therapy. Nat Rev Gastroenterol Hepatol.
2017;14(12):739-49.

57. Embrace the complexity. Nature Immunol. 2009;10(4):325.

58. Nathan C. Points of control in inflammation. Nature. 2002;420(6917):846-52

59. Hernandez-Rocha C, Vande Casteele N. JAK inhibitors: current position in treatment strategies for use in inflammatory bowel disease. Curr Opin Pharmacol. 2020;55:99-109.

60. Loscalzo J et al. (eds.), Network Medicine: Complex Systems in Human Disease and Therapeutics (2017) Harvard University Press: Cambridge, Massachusetts, p.448.

61. Yosef N, Regev A. Writ large: genomic dissection of the effect of cellular environ-ment on immune response. Science. 2016;354(6308):64-8.

62. Rajkomar A et al. Machine learning in medicine. $\mathrm{N}$ Engl $\mathrm{J}$ Med. 2019;380(14):1347-58.

63. Ie Berre $\mathrm{C}$ et al. Application of artificial intelligence to gastroenterology and hepatology. Gastroenterology. 2020;158(1):76-94.

64. Barabási AL et al. Network medicine: a network-based approach to human disease. Nat Rev Genet. 2011;12(1):5668.

65. Fiocchi $\mathrm{C}$ et al. Results of the Seventh Scientific Workshop of ECCO: precision medicine in IBD what, why, and how. J Crohn Colitis. 2021;15(9):1410-30.

66. Yadav A et al. Precision medicine - networks to the rescue. Cur Opin Bio-technol. 2020;63:177-89. 